\title{
Angiomiolipoma renal gigante: una presentación poco frecuente
}

\section{Giant renal angiomyolipoma: a rare presentation Angiomiolipoma gigante renal: uma apresentação rara}

doi) http://dx.doi.org/10.35954/SM2019.38.2.7

Marcelo Alves a (D) https://orcid.org/0000-0003-0057-375X

Ariel Fraga ${ }^{b}$ (D) https://orcid.org/0000-0003-1338-0842

Fabiana Gobertti c

https://orcid.org/0000-0002-6131-640X

Andrea Rocca ${ }^{d}$

https://orcid.org/0000-0002-1316-6734

(a) Servicio de Urología. Hospital Central de las Fuerzas Armadas.

(b) Servicio de Cirugía Pediátrica. Hospital Central de las Fuerzas Armadas.

(c) Servicio Centro de Materiales. Hospital Central de las Fuerzas Armadas.

(d) Servicio de Enfermedades Hepáticas. Hospital Central de las Fuerzas Armadas.

\section{RESUMEN}

El angiomiolipoma renal es un tumor benigno de hallazgo incidental ecográfico en el $60 \%$ de casos. El estudio con Tomografía Computada o Resonancia Magnética hace diagnóstico por la presencia de grasa intratumoral. El calibre vascular aumentado lo distingue en la tomografía del liposarcoma, diagnóstico diferencial cuando el angiomiolipoma es de gran tamaño. De acuerdo a su volumen, se define control con observación o tratamiento activo para evitar la principal complicación: la hemorragia espontánea que se presenta en un 50-60\% de los casos, y puede ser grave. El objetivo es eliminar el tumor con la menor morbilidad preservando función renal. Existen varias opciones a seleccionar según el tamaño del tumor, y la función renal actual o con riesgo de alteración: nefrectomía total; nefrectomía preservadora de nefronaspor cirugía robótica es otra alternativa conservadora en desarrollo; embolización selectiva de ramas de la arteria renal o técnicas de ablación tisular (crioablación, radiofrecuencia). La nefrectomía parcial laparoscópica tiene resultados comparables a la cirugía radical. Presentamos el caso clinico de una paciente con un angiomiolipoma renal gigante y su resolución quirúrgica.

PALABRAS CLAVE: Angiomiolipoma; Nefrectomía.

\begin{abstract}
Renal angiomyolipoma is a benign tumor of incidental ultrasound finding in $60 \%$ of cases. The study with Computed Tomography or Magnetic Resonance makes diagnosis by the presence of intratumoral fat. The increased vascular calibre distinguishes it in liposarcoma tomography, differential diagnosis when the angiomyolipoma is large. According to its volume, control is defined with observation or active treatment to avoid the main complication: spontaneous hemorrhage that occurs in $50-60 \%$ of cases, and can be serious. The objective is to eliminate the tumor with the least morbidity while preserving renal function. There are several options to select according to the size of the tumor, and the current renal function or with risk
\end{abstract}


of alteration: total nephrectomy; nephrone-preserving nephrectomyby robotic surgery is another conservative alternative in development; selective embolization of branches of the renal artery or techniques of tissue ablation (cryoablation, radiofrequency); laparoscopic partial nephrectomy has results comparable to radical surgery.

We present the clinical case of a patient with a giant renal angiomyolipoma and its surgical resolution.

KEY WORDS: Angiomyolipoma; Nephrectomy.

\section{RESUMO}

O angiomiolipoma renal é um tumor benigno com achado ultrassonográfico incidental em $60 \%$ dos casos. O estudo com Tomografia Computadorizada ou Ressonância Magnética faz o diagnóstico pela presença de gordura intratumoral. $\mathrm{O}$ aumento do calibre vascular distingue-o na tomografia de lipossarcoma, diagnóstico diferencial quando o angiomiolipoma é grande. De acordo com seu volume, o controle é definido com observação ou tratamento ativo para evitar a principal complicação: hemorragia espontânea que ocorre em $50-60 \%$ dos casos, podendo ser grave. O objetivo é eliminar o tumor com menor morbidade, preservando a função renal. Existem várias opções para selecionar de acordo com o tamanho do tumor e a função renal atual ou com risco de alteração: a nefrectomia total; a nefrectomia robótica conservadora de nefrectomia é outra alternativa conservadora no desenvolvimento; a embolização seletiva de ramos da artéria renal ou técnicas de ablação tecidual (crioablação, radiofreqüência); a nefrectomia parcial laparoscópica tem resultados comparáveis à cirurgia radical.

Apresentamos o caso clínico de um paciente com angiomiolipoma renal gigante e sua resolução cirúrgica.

PALAVRAS CHAVE: Angiomiolipoma; Nefrectomia.

\section{INTRODUCCIÓN}

Los angiomiolipomas (AML) son tumores mesenquimales generalmente benignos, formados por tejido graso maduro, músculo liso y vasos sanguíneos aberrantes.

Su incidencia es entre $1-3 \%$ de los tumores renales, pero es el más frecuente de los tumores benignos. La etiología y patogenia del AML son desconocidas.

Su detección incidental es creciente por el uso cada vez mayor de técnicas de imagen realizadas por otras causas. Tiene características clínicas y radiológicas específicas que permiten hacer el diagnóstico preoperatorio en la mayoría de los casos. Para pacientes con AMLs menores a $4 \mathrm{~cm}$ y asintomáticos es posible el control clínico y de imagen periódicos. En los casos de mayor volumen el riesgo de sangrado grave es mayor, por lo que se considera el tratamiento activo. El mismo ofrece distintas modalidades terapéuticas: nefrectomía total o parcial, o terapias no resectivas (embolización arterial, radiofrecuencia). La selección se realiza de acuerdo a cada situación particular, con el objetivo de eliminar la masa tumoral con la premisa de preservar la función renal $(1,2)$.

\section{CASO CLÍNICO}

Presentamos el caso de una paciente sexo femenino de 54 años de edad, que consulta por dolor intenso de aparición brusca en hipocondrio derecho irradiado a fosa lumbar derecha, con nauseas y sudoración profusa, sin repercusión hemodinámica y que requiere analgesia endovenosa. Como antecedente relevante presenta dolor lumbar derecho, gravativo, sin irradiaciones, de leve intensidad de dos años de evolución.

Al examen físico tiene un buen estado general y se destaca el hallazgo de una tumoración palpa- 
ble que ocupa el hipocondrio y la fosa lumbar derechos, redondeada, de superficie lisa, indolora, de $10 \times 7 \mathrm{~cm}$, firme, que no sobrepasa la línea media con contacto lumbar interno e inmediato y peloteo. Semiológicamente esta tumoración corresponde a una renomegalia.

La ecografía abdominal y de aparato urinario evidencia una tumoración sólida renal derecha hiper-cogénica de 110 × 90 × 70 mm a nivel mesorrenal que se extiende a polo inferior.

La tomografía computada (TC) tóraco-abdominal con contraste muestra el riñón derecho aumentado de tamaño en forma difusa, a expensas de una tumoración mesorrenal, grande, de aproximadamente $15 \times 9 \mathrm{~cm}$, heterogénea que se extiende hacia el polo inferior y el retroperitoneo. Esta tumoración tiene densidad grasa $(-30 \mathrm{UH})$ y se realza levemente con la administración de contraste $\mathrm{i} / \mathrm{v}$, con un umbral de atenuación entre 20 y $100 \mathrm{UH}$ (figura 1). Otro elemento a destacar es la importante circulación colateral que presenta la lesión. Estas características radiológicas permiten plantear el diagnóstico clínico, tomográfico y etiológico de un angiomiolipoma (AML) gigante ya que es el único tumor renal que tiene grasa.

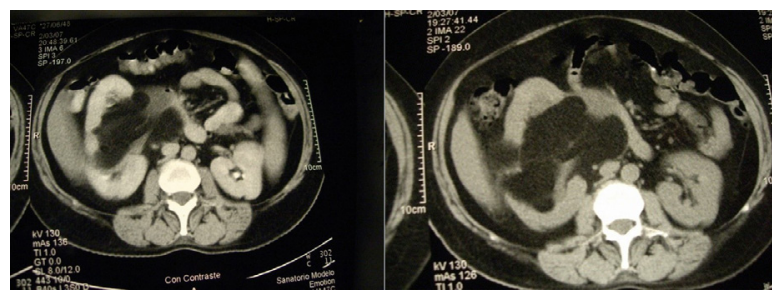

Figura 1. TC Angiomiolipoma Renal Derecho.

Se realiza cirugía de coordinación por abordaje abierto transperitoneal mediante incisión transversa de hipocondrio derecho extendida a flanco, con dominio primario del pedículo renal. Los hallazgos intraoperatorios confirman un tumor con gran circulación colateral, de contenido predominantemente graso, topografiado a nivel del riñón derecho, con un claro plano de separación con tejidos y órganos vecinos. Procediéndose a la nefrectomía radical en forma reglada y sin incidentes. Se envía pieza a estudio anátomo patológico. La evolución postoperatoria es buena y sin complicaciones. La anatomía patológica informa: Macroscopía: Riñón derecho mide 180 × 14 × 90 mm. Se observa una tumoración que lo atraviesa a nivel del hilio y polo inferior de aspecto adiposo, infiltrante, de $16 \times 11 \times 8 \mathrm{~mm}$, que deja un remanente de parénquima renal de $50 \mathrm{~mm}$ de eje mayor, obliterando el hilio renal (figura 2).

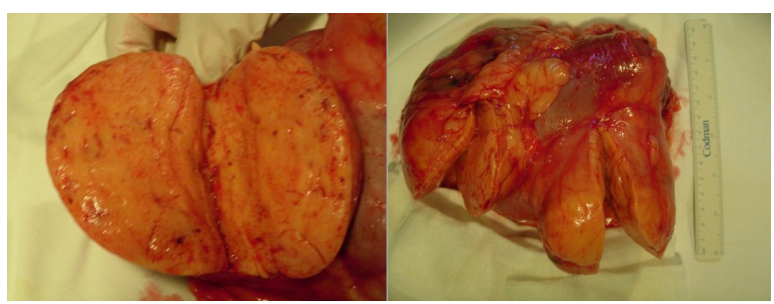

Figura 2. Tumoración renal. Macroscopía.

La microscopía observa una proliferación celular de estirpe mesenquimática constituida por adipocitos maduros y células musculares lisas. Los vasos son de aspecto dismórfico, de paredes engrosadas y con luces excéntricas (figura 3). La lesión presenta un crecimiento expansivo infiltrativo con compromiso del hilio renal. Todas estas características son concordantes con el diagnóstico de un angiomiolipoma renal gigante.
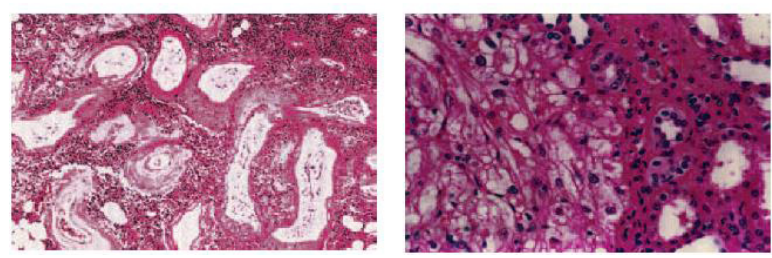

Figura 3. Microscopía AML.

\section{DISCUSIÓN}

La presentación clínica más frecuente del AML renal es el hallazgo incidental en un estudio por imágenes de una lesión pequeña y asintomática $(60 \%$ de los casos). La mayoría de las lesiones no superan los $5 \mathrm{~cm}$ de diámetro. Si las lesiones son grandes pueden ser sintomáticas presentándose con: dolor en flanco $(40 \%)$, tumoración palpable $(40 \%)$, hematuria $(30 \%)$ y shock hipovolémico (10\%). En algunos casos se presenta con la tríada de Lenk caracterizada por dolor agudo, masa palpa- 
ble y signos de hemorragia interna (shock hipovolémico), que requiere nefrectomía de urgencia (1). La principal complicación de los AML, que puede ser de extrema gravedad, es el sangrado. La existencia de vasos anormales dentro de la lesión (aneurismas) explica la predisposición a las hemorragias dentro del tumor y en el retroperitoneo. Los AML mayores de $4 \mathrm{~cm}$ pueden presentar hemorragias espontáneas en un $50-60 \%$ de los casos (2). En el caso de esta paciente se destaca el tamaño del AML, su presentación con dolor y tumoración palpable a pesar de lo cual no se detectó hemorragia tumoral como complicación.

El diagnóstico de AML renal es imagenológico por TC o Resonancia Magnética (RM) y se basa en la presencia de grasa en el tumor. Con excepción del lipoma perirrenal y del liposarcoma, la presencia de grasa en un tumor renal no calcificado, es altamente específico de AML.

La ecografía abdominal ha incrementado la detección de los tumores renales asintomáticos, siendo el angiomiolipoma y el carcinoma renal los más frecuentemente detectados. El angiomiolipoma se ve como una masa hiperecogénica bien delimitada. Esta hiperecogenicidad se debe a la interface acústica entre el tejido graso y el músculo liso, lo cual hace del angiomiolipoma el más ecodenso de los tumores renales. Pueden presentar áreas hipoecogénicas en caso de necrosis o hemorragia antigua. Tanto el carcinoma de células renales (CCR) como el AML pueden presentar características ecográficas similares. Las lesiones renales ecogénicas deben estudiarse en forma complementaria con TC o RM a fin de definir la presencia de grasa intratumoral. Es excepcional la presencia de grasa en el CCR (3).

En la TC el AML se presenta como un tumor heterogéneo, con contenido de densidad grasa y de partes blandas, que incrementa en forma variable con el contraste de acuerdo a su contenido de músculo liso y vasos. La detección de pequeñas cantidades de grasa requiere un criterio diagnóstico estricto, con un umbral de atenuación de menos $20 \mathrm{UH}$ o menor.
Dado que el angiomiolipoma puede crecer exofíticamente, es frecuente su extensión al espacio perirrenal, quedando siempre un pequeño defecto en el parénquima renal en su lugar de origen. La demostración de una muesca en el parénquima renal, la presencia de vasos dilatados o aneurismáticos y la multiplicidad de las lesiones colaboran en el diagnóstico tomográfico de $\operatorname{AML}(3,4)$. Si la TC no es diagnóstica, se debe recurrir a la RM, utilizando secuencias dinámicas con contraste y técnica de supresión grasa (3). La resonancia magnética (RM) está indicada en aquellos casos en los cuales existe la duda de la presencia de grasa intratumoral ya que ésta puede ponerse en evidencia con la técnica de supresión grasa, o cuando la TC con contraste intravenoso estuviera contraindicada. Otras indicaciones de la RM son un tumor voluminoso y/o con trombo en la vena renal o cava en que los cortes sagitales permiten el diagnóstico de extensión y relaciones con órganos vecinos. El diagnóstico diferencial de un AML renal cuando posee gran tamaño es con el liposarcoma. Ambas son masas retroperitoneales con contenido graso. En ocasiones, sus apariencias son muy similares, e inclusive su diagnóstico histológico presenta dificultades. Sin embargo, su diferenciación es importante ya que el pronóstico y tratamiento son diferentes.

Comparativamente, el liposarcoma nace en la grasa retroperitoneal, incluyendo la grasa perirrenal, dentro de la fascia de Gerota, asociados frecuentemente a la cápsula renal y por ello llamados liposarcomas capsulares. Cuando crecen desplazan, comprimen y distorsionan el riñón, pero no invaden el parénquima renal adyacente. Por otra parte, el angiomiolipoma contiene vasos aumentados de tamaño, que sólo pueden ser vistos en las TC contrastadas, mientras que los liposarcomas bien diferenciados son relativamente avasculares y los vasos presentes no están agrandados, siendo éste un signo auxiliar para el diagnóstico. También debe considerase como signo auxiliar el hecho de la multiplicidad de lesiones encontradas en el AML (4). 
Otros diagnósticos diferenciales lo constituyen el CCR (que en muy raras ocasiones pueden contener grasa) y también se han reportado casos aislados de metástasis con contenido graso.

El AML tiene en la mayoría de los casos un comportamiento benigno, sin embargo, en algunos casos se producen complicaciones potencialmente graves como la hemorragia retroperitoneal. Ésta es más frecuente en pacientes con tumores mayores a $4 \mathrm{~cm}$ o embarazadas. En casos poco frecuentes un AML puede presentar: trombo tumoral en la vena renal y/o vena cava; multifocalidad e incluso la afectación de ganglios linfáticos regionales. Esta forma de presentación corresponde a un patrón de crecimiento multifocal más que metástasis. Es excepcional que se desarrolle un sarcoma a partir de un AML esporádico. Con mayor frecuencia se han comunicado casos de transformación epitelioide de un AML clásico. Únicamente las metástasis o un franco crecimiento sarcomatoso de alto grado pueden ser considerados criterios estrictos de malignidad $(2,3)$.

La anatomía patológica describe al AML macroscópicamente como un tumor bien delimitado, no encapsulado. Generalmente tiene un crecimiento expansivo más que infiltrativo, con claro plano de separación con el riñón. Este tipo de tumor en la mayoría de los casos se desarrolla dentro del parénquima renal y es exofítico en un $25 \%$ de los casos. En el $88 \%$ de los casos se extiende a través de la cápsula renal y con escasa frecuencia al sistema venoso. El AML clásico está compuesto por una proporción variable de tejido adiposo, células musculares fusiformes, epiteloides y vasos sanguíneos anómalos de paredes gruesas. En general los pacientes con AML menores de $4 \mathrm{~cm}$ y asintomáticos pueden ser controlados (sin un tratamiento activo) con observación y evaluaciones repetidas con ecografía y TC cada 6 a 12 meses para determinar la velocidad de crecimiento y la importancia clínica de la lesión. La mayoría de los investigadores sugieren $4 \mathrm{~cm}$ como el tamaño umbral de intervención, aunque otros sugieren $8 \mathrm{~cm}$. En el caso de pacientes con tumores de mayor tamaño y/o sintomáticos, debe considerarse un tratamiento activo teniendo presente la edad, patologías coexistentes y otros factores relacionados. El objetivo terapéutico es eliminar el tumor con la menor morbilidad preservando la función renal. Existen varias opciones terapéuticas: técnicas resectivas (abierta o video asistida) como la nefrectomía total o nefrectomía preservadora de nefronas (NPN) y no resectivas como la embolización selectiva de ramas de la arteria renal (SAE) o técnicas de ablación tisular (crioablación, radiofrecuencia). Actualmente el tratamiento en lo posible debe ser conservador. En especial los pacientes con enfermedad bilateral, insuficiencia renal previa u otros trastornos con riesgo de deterioro funcional renal en el largo plazo son candidatos a técnicas conservadoras $(5,6)$.

La nefrectomía se realiza en casos de diagnóstico no definido, asociación con CCR o sustitución de la mayor parte del parénquima renal por tumor. La mayoría de los pacientes con una hemorragia aguda o potencialmente fatal requieren una nefrectomía total de urgencia. El abordaje para el tratamiento quirúrgico puede ser abierto, video asistido (laparoscópia, lumboscópica) o robótico. La evolución de la laparoscopia ha permitido que la nefrectomía parcial laparoscópica sea una alternativa en los tumores de entre 4 a $7 \mathrm{~cm}$ y en localizaciones complejas, con resultados comparables a la cirugía radical (7).

En la actualidad hay trabajos que establecen que la NPN robótica es una opción conservadora efectiva, segura, que brinda buena preservación de la función renal en seguimiento a largo plazo $(7,8)$. La SAE puede estar indicada cuando los tumores son más pequeños y tienen menor cantidad de angiogénesis, dando mejores resultados con menos complicaciones $(2,3)$.

La radiofrecuencia (RFA) parece ser una prometedora estrategia alternativa. Tiene la ventaja potencial de la repetitividad. En los estudios actuales, la RFA no dio lugar a complicaciones mayores a largo plazo y presentó una reducción de la masa tumoral del $20 \%$. El atractivo es la selectividad 
de la zona de acción, evitando parénquima renal normal y disminuyendo el riesgo de sangrado (6). Actualmente se están investigando nuevos fármacos como inhibidores de la angiogénesis (sirolimus) que podrían ser útiles y evitarían las terapias activas si se administran precozmente en el proceso de la enfermedad.

In-vitro los estudios que utilizan inmunoblot e inmunohistoquímica han confirmado la activación de la vía mTOR en el AML esporádico. Por lo tanto, los inhibidores de la mTOR, como el sirolimus podrían tener un papel en el tratamiento $(2,5,9)$.

DECLARACIÓN DE CONFLICTOS DE INTERESES: Los autores no reportan ningún conflicto de interés. El estudio se realizó con recursos propios de los autores y/o la institución a la que representan.

\section{REFERENCIAS}

(1) Pedemonte JG, Degiovanni D, Pusterla D, Reibel C, Di Nucci J, Boccio C, et al.

Tríada de Lenk como presentación clínica del angiomiolipoma.

Actas Urol Esp 2008; 32(8):850-854.

(2) Bestard Vallejo JE, Trilla Herrera E, Celma Domenech A, Pérez Lafuente M, de Torres Ramírez I, Morote Robles J. Angiomiolipomas renales: presentación, tratamiento y resultado de 20 casos. Actas Urol Esp 2008; 32(3):307-315.

(3) Bissler JJ, Kingswood JC.

Renal angiomyolipomata.

Kidney Int 2004; 66(3):924-934.

doi: 10.1111/j.1523-1755.2004.00838.x

(4) Henske EP, Ao X, Short MP, Greenberg R, Neumann HP, Kwiatkowski DJ, et al. Frequent progesterone receptor immunoreactivity in tuberous sclerosis-associated renal angiomyolipomas. Mod Pathol 1998; 11(7):665-668.
(5) Sooriakumaran P, Gibbs P, Coughlin G, Attard V, Elmslie F, Kingswood C, et al. Angiomyolipomata: challenges, solutions, and future prospects based on over 100 cases treated. BJU Int 2010; 105(1):101-6. doi: 10.1111/j.1464-410X.2009.08649.x

(6) Iriarte Soldevilla J, Senarriaga Ruiz de la Illa N, Lacasa Viscasillas I, Loizaga Iriarte A, Zubiaur Libano C, Unda Urzaiz M.

Tratamiento actual de las masas renales pequeñas. Actas Urol Esp 2009; 33(5):505-51.

(7) Castillo O, Fonerón A, Vidal I, Tapia C.

Nefrectomía parcial laparoscópica en angiomiolipoma gigante. Rev Chil Cir 2008; 60(5):442-446. doi: 10.4067/S0718-40262008000500014

(8) Lin CY, Yang CK, Ou YC, Chiu KY, Cheng $\mathrm{CL}, \mathrm{Ho} \mathrm{HC}$. Long-term outcome of robotic partial nephrectomy for renal angiomyolipoma.

Asian J Surg 2018; 41(2):187-191.

doi: 10.1016/j.asjsur.2016.11.003

(9) El-Hashemite N, Walker V, Zhang H, Kwiatkowski DJ. Loss of Tsc1 or Tsc2 induces vascular endothelial growth factor production through mammalian target of rapamycin. Cancer Res 2003; 63(17):5173-7. 